\title{
Peculiarity of Zr in the Neutron Absorption Cross-section and Corrosion Resistance in Water
}

\author{
Yoshiharu Mae \\ Maetech, Mimuro, Midori Ward, Saitama City, Japan \\ Email address: \\ yoshimae@hotmail.com \\ To cite this article: \\ Yoshiharu Mae. Peculiarity of $\mathrm{Zr}$ in the Neutron Absorption Cross-section and Corrosion Resistance in Water. International Journal of \\ Materials Science and Applications. Vol. 6, No. 5, 2017, pp. 235-240. doi: 10.11648/j.ijmsa.20170605.12
}

Received: August 2, 2017; Accepted: August 15, 2017; Published: August 28, 2017

\begin{abstract}
The elements $\mathrm{Be}, \mathrm{Bi}, \mathrm{Mg}, \mathrm{Pb}, \mathrm{Zr}, \mathrm{Al}, \mathrm{Ca}, \mathrm{Na}, \mathrm{Sn}, \mathrm{Rb}$ and $\mathrm{Ce}$ are the metallic elements of small thermal neutron absorption cross section. Except $\mathrm{Be}$ and $\mathrm{Zr}$, they are all soft metals. But the reasons for these small thermal neutron absorption cross-sections are not known. To clarify its mechanism, the thermal neutron absorption cross-sections of elements were plotted on the TC-YM diagram. They lie on a line connecting the elements of low Young's modulus on the TC-YM diagram. The author at first considered that the neutron absorption characteristics of elements relate to the neutron multiple number which means the number of neutrons per proton in the nucleus. The absorption cross-section of elements roughly increases with increasing neutron multiple number. Among the elements of small neutron absorption cross section, only $\mathrm{Zr}$ and Be are the elements of high melting temperature. $\mathrm{Zr}$ should not show the small neutron absorption cross section inherently. But in fact, $\mathrm{Zr}$ is exceptional both on the TC-YM diagram and in the neutron multiple number. $\mathrm{Zr}$ has a small absorption cross-section against the general trends in both the TC-YM diagram and neutron multiple number. On the other hand, the corrosion resistance of Zircaloy is given by the anodic protection provided by the precipitates of metallic compounds containing $\mathrm{Fe}$ and $\mathrm{Cr}$. It is fortunate that $\mathrm{Fe}$ and $\mathrm{Cr}$ belong to the element group of the metals nobler than $\mathrm{Zr}$ and that of no solubility in $\mathrm{Zr}$ on the TC-YM diagram.
\end{abstract}

Keywords: Thermal Neutron Absorption Cross Section, Neutron, Proton, Young's Modulus, Thermal Conductivity, Anodic Protection, $\mathrm{Zr}$

\section{Introduction}

Zircaloy has been used widely as a cladding material in water reactors due to its small thermal neutron absorption cross-section, its good corrosion resistance to high temperature water and good mechanical properties. These excellent properties of Zircaloy seem to be self-evident, but some story must be hidden behind them. Formerly, the author showed that the corrosion resistance of Zircaloy was provided by the precipitates in the alloy matrix through the mechanism of the anodic protection [1]. Recently, the author introduced the diagram with thermal conductivity on the abscissa and Young's modulus on the ordinate to explain the various properties of elements [2-6]. This diagram will be called "TC-YM diagram" or "diagram". Using this diagram, the mechanism of the small thermal neutron absorption cross-section of $\mathrm{Zr}$ and the mechanism of corrosion resistance of Zircaloy were studied and are reported here.

\section{Thermal Neutron Absorption Cross-Section of Elements}

The data of the thermal neutron absorption cross-section were taken from the old data of JAERI (Japan Atomic Energy Research Institute) report in 1962 [7]. Hereafter, the thermal neutron absorption cross-section is referred to simply as "the absorption cross-section".

Figure 1 shows the distribution of the absorption cross-section of elements on the TC-YM diagram. The figures in the legend show the range of the absorption cross-section of elements in the logarithmic scale of barn.

Figure 1 shows that the elements of small absorption cross-section are located at the low Young's modulus range and from small thermal conductivity up to medium range. This is quite a new pattern. It is different from any other patterns obtained for the properties of elements so far. In the medium 
thermal conductivity range, the element such as Be has small absorption cross-section in spite of its high Young's modulus.

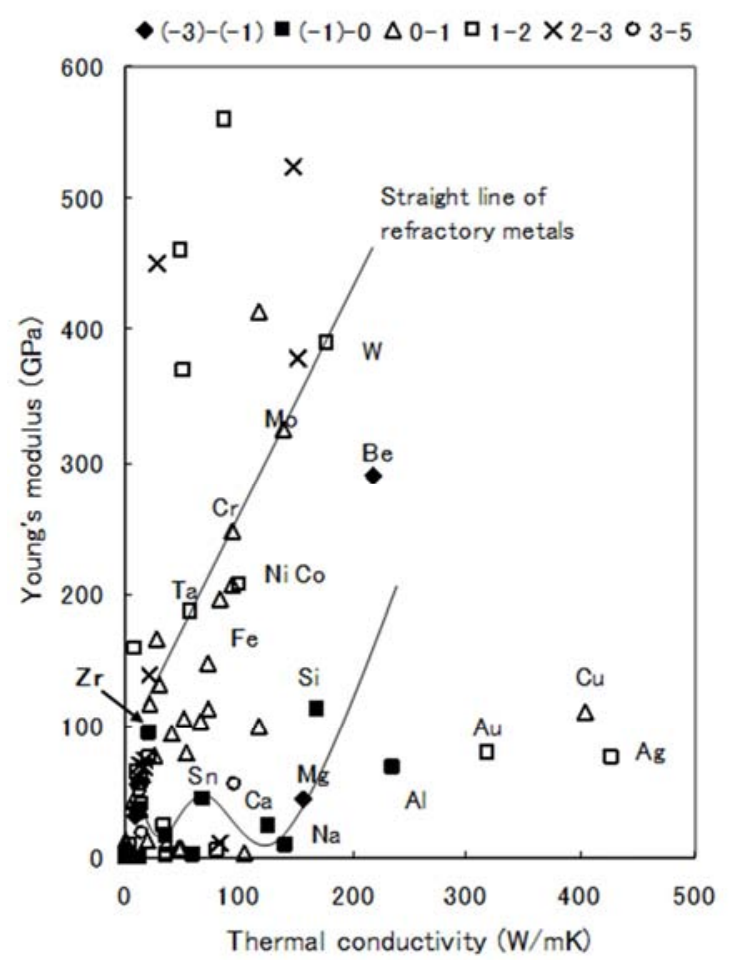

Figure 1. Absorption cross-section of elements on the TC-YM diagram.

Figure 2 shows the area of small thermal conductivity and small Young's modulus of Figure 1.

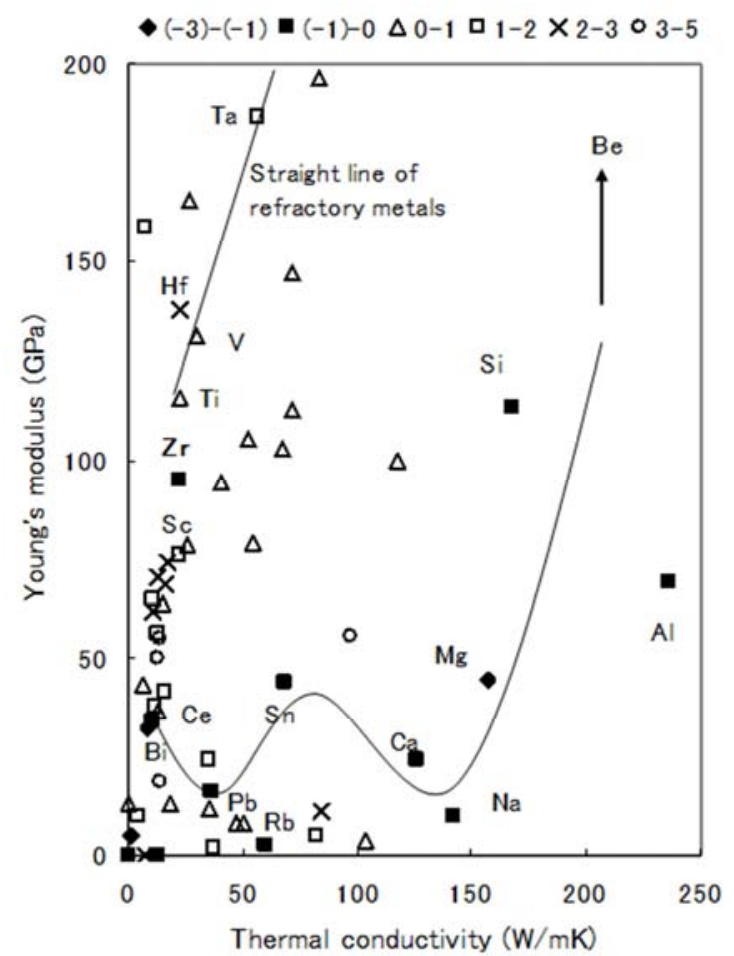

Figure 2. Absorption cross-section of elements with low Young's modulus and low thermal conductivity on the diagram.
The elements of small absorption cross-section lie on a curve connecting $\mathrm{Bi}, \mathrm{Ce}, \mathrm{Pb}, \mathrm{Sn}, \mathrm{Ca}, \mathrm{Na}, \mathrm{Mg}, \mathrm{Si}, \mathrm{Be}$ and $\mathrm{Al}$. They are all low melting temperature elements except Be and Si. Therefore, they are not suitable for the fuel cladding in the water reactor. $\mathrm{Zr}$ is located far from these elements, and surprisingly it shows low absorption cross-section. As shown in Figure 2, $\mathrm{Zr}$ is perfectly surrounded by the elements of large absorption cross-section. In this point of view, $\mathrm{Zr}$ is an exception in the distribution of elements on the TC-YM diagram.

Figure 3 shows the distribution of the absorption cross-section of elements on the periodic table.

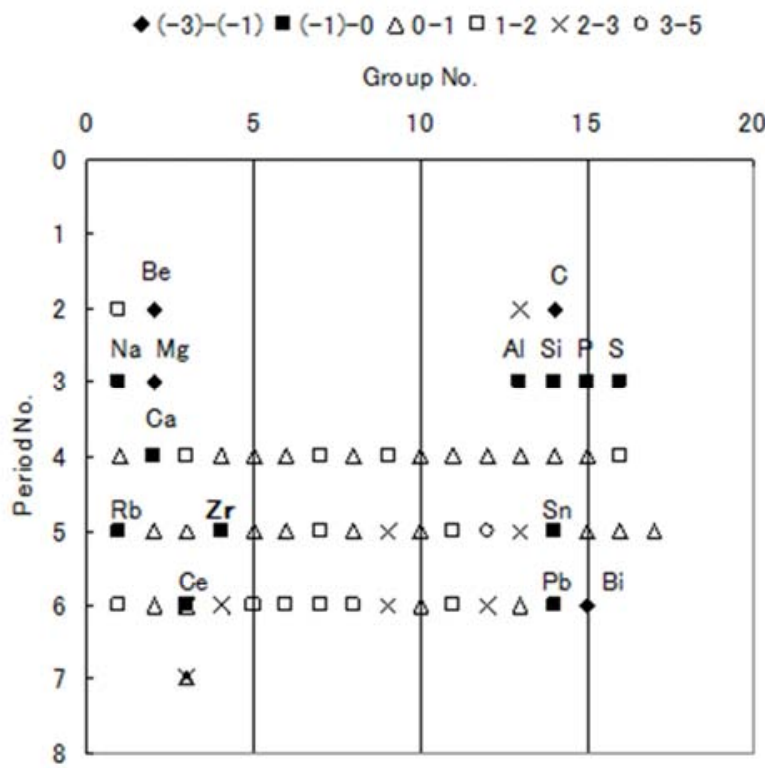

Figure 3. Absorption cross-section of elements displayed on the periodic table.

All the elements in the $3^{\text {rd }}$ period are the elements of small absorption cross-section. The transition elements show large absorption cross-section except $\mathrm{Zr}$. $\mathrm{Zr}$ is the only elements of small absorption cross-section among the transition metals.

\section{The Effect of the Neutron Multiple Number on the Absorption Cross-Section of Elements}

At first, the author speculated that the elements which have relatively much neutrons to protons in each nucleus are not apt to absorb neutrons. The author defined the "neutron multiple number" as the quotient when the number of neutrons is divided by the number of protons in the nucleus [5]. Figure 4 shows the neutron multiple number of elements on the diagram.

The neutron multiple number varies from nearly 1 to nearly 1.6 for solid elements. The figures in the legend show the range of the neutron multiple number. 


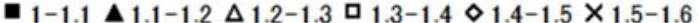

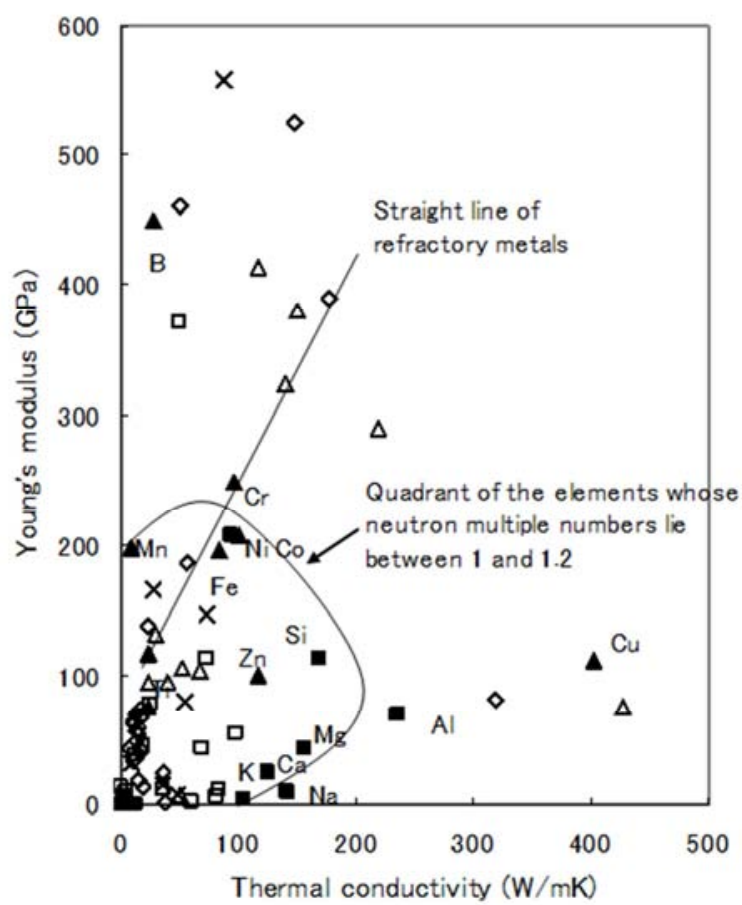

Figure 4. Neutron multiple number of elements on the diagram.

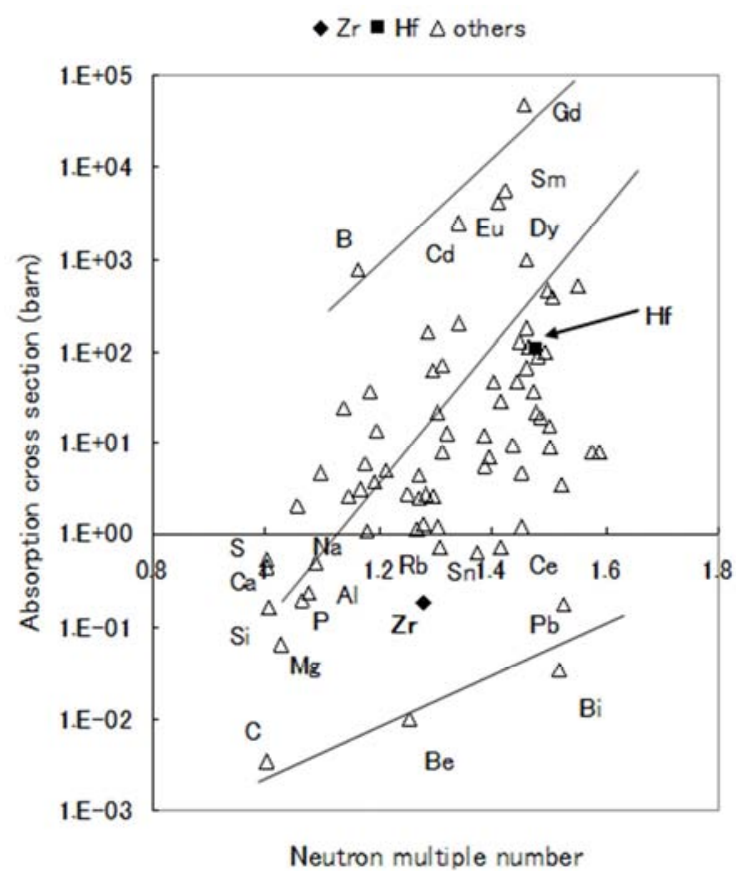

Figure 5. Correlation between the absorption cross-section and the neutron multiple number of elements.

Surprisingly, the neutron multiple number of elements also draws a pattern on the diagram. The elements of the smallest neutron multiple numbers form a quadrant on the diagram. This corresponds to the abundance of elements in the universe [5], but it doesn't seem to correspond to the absorption cross-section shown in Figure 1. The author thought at first that the elements of small absorption cross-section would have large neutron multiple numbers and be saturated with neutrons. If so, the elements of small neutron multiple numbers should be located outside or inside the quadrant on the diagram. But in fact, among the elements of small absorption cross-section, $\mathrm{Bi}, \mathrm{Ce}, \mathrm{Pb}$ and $\mathrm{Sn}$ are located inside the quadrant, $\mathrm{Be}$ is outside the quadrant, and $\mathrm{Ca}, \mathrm{Na}, \mathrm{Mg}, \mathrm{Si}$ and $\mathrm{Al}$ are included in the quadrant. Therefore, the correlation between the neutron multiple number and the absorption cross-section could not be expected. Nevertheless, it was done for confirmation. Results are shown in Figure 5.

The correlation between both factors is complicated. The elements of small absorption cross-section are located at the lower part of the figure. The elements $\mathrm{C}, \mathrm{Be}, \mathrm{Bi}$ and $\mathrm{Pb}$ show exceptionally small absorption cross-section.

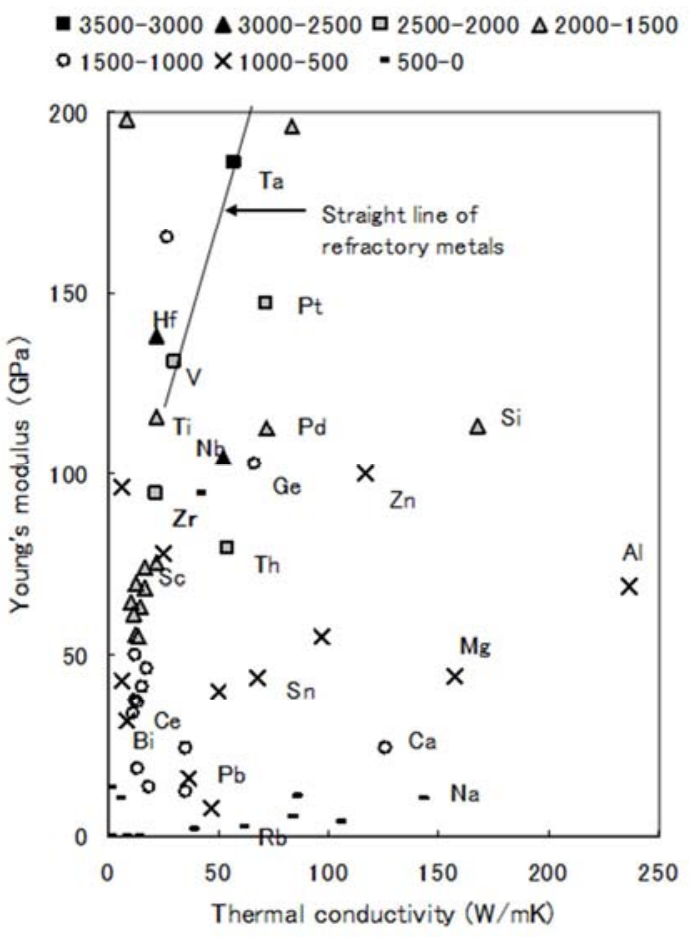

Figure 6. Melting temperatures of elements on the diagram.

The elements of large absorption cross-section B, Cd, Eu, $\mathrm{Sm}$ and $\mathrm{Gd}$ are located at the upper part of the figure.

Except these elements, a vague tendency can be seen. With large scatter, the absorption cross-section increases as the neutron multiple number increases. The elements of small absorption cross-section $\mathrm{Mg}, \mathrm{Si}, \mathrm{P}, \mathrm{Al}, \mathrm{Ca}, \mathrm{S}$ and $\mathrm{Na}$ have small neutron multiple numbers.

In contrast to this, $\mathrm{Zr}$ does not follow these trends. $\mathrm{Zr}$ is isolated from them and surrounded with the elements of large absorption cross-section on the TC-YM diagram, as shown in Figure 2. Moreover, $\mathrm{Zr}$ is located at the position of medium neutron multiple number and small absorption cross-section against the general trend of other elements, as shown in Figure 5. These facts show that $\mathrm{Zr}$ is an exception among the elements of low absorption cross-section. $\mathrm{Zr}$ is not a soft metal like other elements of small absorption cross-section. Figure 6 shows the distribution of the melting temperature of elements on the 
diagram in the same area as in Figure 2. The figures in the legend show the temperature range of the melting temperature in $\mathrm{K} . \mathrm{Zr}$ is a high melting temperature element at the downstream of Ta, V and Ti, connecting to Sc. Consequently, it is known that $\mathrm{Zr}$ is the only element applicable for the cladding material of the nuclear fuel in the water reactor.

\section{The Factors Affecting the Corrosion Resistance of Zircaloy}

Zircaloy is an alloy developed for the cladding material of nuclear fuel in the water reactor. Its nominal chemical composition is $\mathrm{Zr}-1.3$ mass\% $\%$ Sn- 0.1 mass\% Fe- 0.1 mass\% $\mathrm{Cr}$. It is said that $\mathrm{Sn}$ is added for the solution hardening and $\mathrm{Fe}$ and $\mathrm{Cr}$ are added for the corrosion resistance. Formerly, the author clarified that the substance of corrosion resistance of Zircaloy is the anodic protection provided by the precipitates of $\mathrm{Zr}(\mathrm{FeCr})_{2}$ in the $\mathrm{Zr}$ matrix [1]. The precipitates of $\mathrm{Zr}(\mathrm{FeCr})_{2}$ is nobler than the $\mathrm{Zr}$ matrix and enhance the formation of tough oxide film on the $\mathrm{Zr}$ matrix. For that purpose, $\mathrm{Fe}$ and $\mathrm{Cr}$ must be nobler than $\mathrm{Zr}$ and their solubility must be small in $\mathrm{Zr}$. Here, electrochemical properties of $\mathrm{Zr}$ compared to other elements and solubility of elements in $\mathrm{Zr}$ were studied.

\subsection{Standard Electrode Potentials of Elements}

Figure 7 shows the distribution of the standard electrode potentials of elements on the diagram.

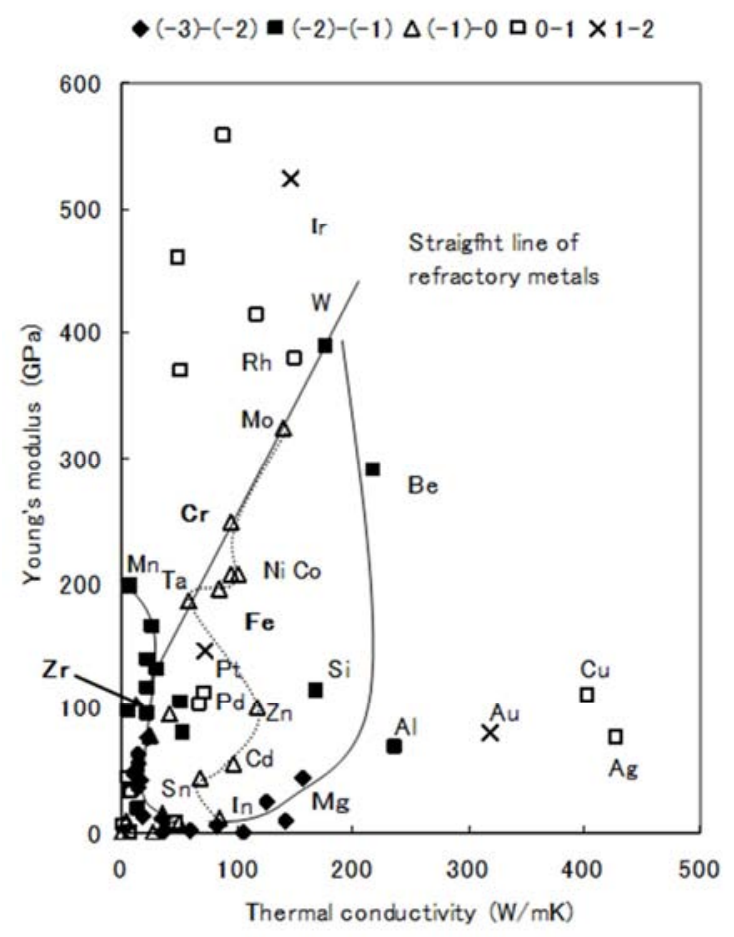

Figure 7. Electrode potentials of elements on the TC-YM diagram.

The figures in the legend show the range of the standard electrode potential of elements in volt. The elements of smallest electrode potentials start from $\mathrm{Mn}$, descend to the bottom of the diagram, creep along the abscissa, and finally rise from $\mathrm{Mg}$ to $\mathrm{W}$. $\mathrm{Zr}$ is included in this element group.

The elements of medium electrode potentials lie on the vertical line at the medium thermal conductivity, and are surrounded by the elements of the smallest electrode potentials. Fortunately, $\mathrm{Fe}$ and $\mathrm{Cr}$ are included in this element group of the medium electrode potentials.

\subsection{Solubility of Elements in $\mathrm{Zr}$}

Formerly, the author showed that the elements with the same crystal structures as the matrix metal and those in the neighborhood of the matrix metal on the TC-YM diagram have large solubility in the matrix metal [2]. Figure 8 shows the distribution of solubility of elements in the â-phase of $\mathrm{Zr}$ on the diagram. The maximum solubility of elements in the â-phase of $\mathrm{Zr}$ at high temperature was adopted as the solubility in the â-phase.

The figures in the legend show the solubility range in $\mathrm{mol} \%$. The elements with the bcc structure and near $\mathrm{Zr}$ have unlimited solubility. The elements showing small amount of solubility lie on a line starting from $\mathrm{Ag}$ and ending at Os. Fe and $\mathrm{Cr}$ are included in this line. The elements of higher thermal conductivity and higher Young's modulus than $\mathrm{Zr}$ show generally low solubility.

Figure 9 shows the distribution of the solubility of elements in the á-phase of $\mathrm{Zr}$ on the diagram. The maximum solubility of elements in the á-phase of $\mathrm{Zr}$ at high temperature was adopted as the solubility in the á-phase of $\mathrm{Zr}$.

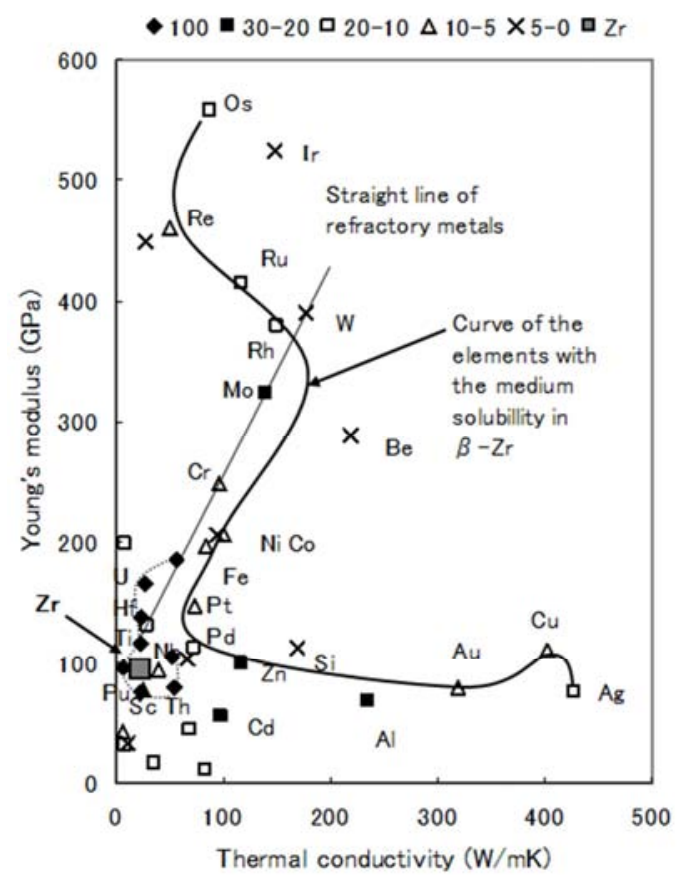

Figure 8. Solubility of elements in the a-phase of $\mathrm{Zr}$ on the TC-YM diagram.

The elements with the hcp structure and near $\mathrm{Zr}$ show the unlimited solubility. The elements of no solubility lie on a line starting from $\mathrm{Th}$ and ending at $\mathrm{B}$. Fe and $\mathrm{Cr}$ are included in this line. The elements of higher thermal conductivity and 
higher Young's modulus than $\mathrm{Zr}$ show low solubility in á-Zr, as in $\hat{a}-\mathrm{Zr}$.

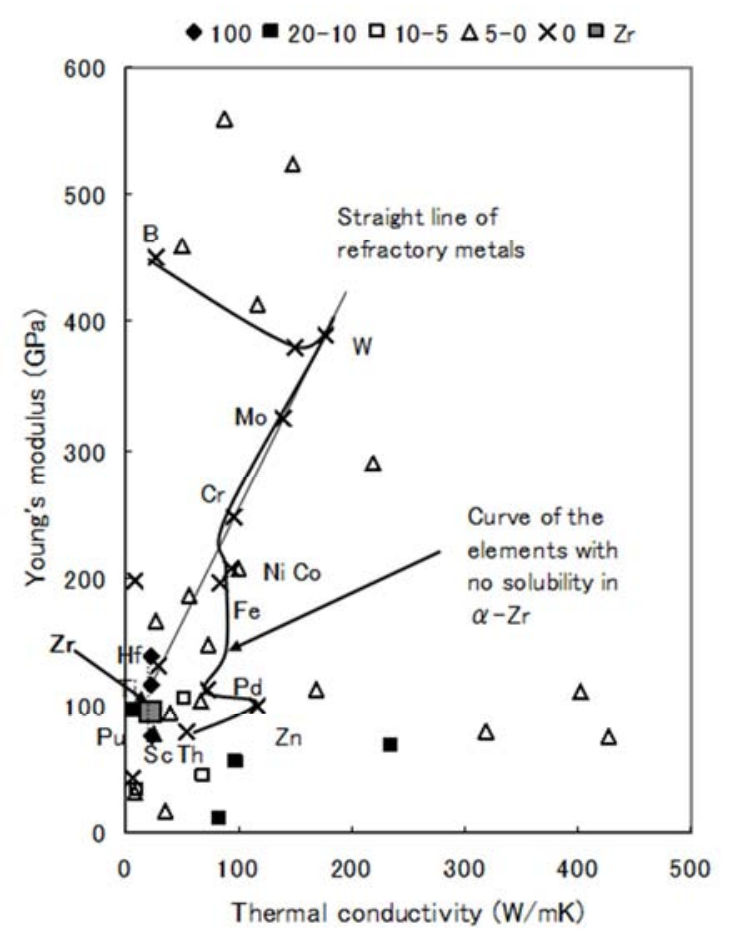

Figure 9. Solubility of elements in the á-phase of Zr on the TC-YM diagram.

\section{Discussion}

\subsection{Thermal Neutron Absorption Cross-Section}

As shown in Figure 5, at most of the elements except $\mathrm{C}, \mathrm{Be}$, $\mathrm{Bi}$ and $\mathrm{Pb}$, with increasing neutron multiple number, the absorption cross-section of elements increases. $\mathrm{Zr}$ is located at the position of small absorption cross-section, off this trend.

$\mathrm{Zr}$ is also isolated on the TC-YM diagram. Figure 2 shows that $\mathrm{Zr}$ is isolated from other elements of small absorption cross-section. The elements in the same $4^{\text {th }}$ group of the periodic table $\mathrm{Ti}, \mathrm{Zr}$ and $\mathrm{Hf}$ lie vertically under the lower end of the straight line of refractory metals. The absorption cross-section is mostly the only difference between them. $\mathrm{Zr}$ has much smaller absorption cross-section than expected from both its position on the diagram and its neutron multiple number, as shown in Figure 2 and 5, respectively. As if it would compensate the small absorption cross-section of $\mathrm{Zr}$, Hf has large absorption cross-section. The large absorption cross-section of $\mathrm{Hf}$ is understandable, because it has large neutron multiple number and follows the trend of the other elements, as shown in Figure 5. But the small absorption cross-section of $\mathrm{Zr}$ can not be explained by its neutron multiple number. The absorption cross-section of $\mathrm{Zr}$ seems to be lowered intentionally by the unknown mechanism.

Reference [8] says only that the desired properties of $\mathrm{Zr}$ alloys are a low neutron-capture cross-section and resistance to corrosion. Reference [9] says that $\mathrm{Zr}$ is used as the nuclear material due to its small thermal neutron absorption cross-section, but Hf must be removed, because Hf has a thousand times larger absorption cross-section. Reference [10] says that $\mathrm{Zr}$ has the smallest thermal neutron absorption cross-section among the metallic elements and superior corrosion resistance and mechanical properties, therefore, it is used as the reactor materials. But none of them mention the mechanism of the small absorption cross-section of $\mathrm{Zr}$. Reference [11] does not mention the relationship between the neutron total cross-section and the inner structure of the nucleus, either.

\subsection{Electrode Potentials and Solubility of Elements in $\mathrm{Zr}$}

For the anodic protection, it is necessary that the alloying elements are nobler than the matrix metal and precipitate at small amount of addition. The nobler elements than $\mathrm{Zr}$ are $\mathrm{Cr}$, Ta, Fe, Ni, Mo and Sn, as shown in Figure 7. Among them, the elements of no solubility in á-Zr are $\mathrm{Cr}, \mathrm{Fe}, \mathrm{Ni}$ and Mo.

It is fortunate that the present alloying elements in Zircaloy, $\mathrm{Fe}$ and $\mathrm{Cr}$ are included in the element group of high electrode potential and no solubility in the á-phase of $\mathrm{Zr}$.

Moreover, $\mathrm{Fe}$ is an inevitable impurity in $\mathrm{Zr}$. $\mathrm{Zr}$ is smelted by Kroll process. Fe is included in $\mathrm{Zr}$ during smelting. It is fortunate that $\mathrm{Fe}$ is a useful element in $\mathrm{Zr}$.

\section{Conclusion}

The low Young's modulus is a necessary condition for the elements of small absorption cross-section.

As a second condition, the neutron multiple number must be small.

$\mathrm{Zr}$ is an exception at both conditions. $\mathrm{Zr}$ is a hard metal with high melting temperature, and its neutron multiple number is large. Nevertheless, Zr has small absorption cross-section.

The corrosion resistance of Zircaloy is given by the anodic protection of the precipitates such as $\mathrm{Zr}(\mathrm{FeCr})_{2}$. It is fortunate that the minor alloying elements $\mathrm{Fe}$ and $\mathrm{Cr}$ are included in the element group of both the high electrode potential and no solubility in á- $\mathrm{Zr}$, as shown on the diagram.

\section{References}

[1] T. Isobe, T, Murai, and Y. Mae, "Anodic protection provided by precipitates in aqueous corrosion of Zircaloy," Zirconium in the nuclear industry: $11^{\text {th }}$ Symposium, ASTM, pp. 203-216, 1999.

[2] Y. Mae, "What the Darken-Gurry plot means about the solubility of elements in metals," Metall. Mater. Trans. A, vol. 47, pp. 6498-6506, Dec. 2016.

[3] Y. Mae, "Anthropic principle observed in the material properties of Fe,” J. Mater. Sci. Res., vol. 6, No. 3, pp. 11-19, 2017.

[4] Y. Mae, "Schematic Interpretation of anomalies in the Physical Properties of Eu and $\mathrm{Yb}$ among the lanthanides," Int. J. Mater. Sci. App., vol. 6, pp. 165-170, 2017.

[5] Y. Mae, "Neutron multiple number as a factor ruling both the abundance and some material properties of elements," J. Mater. Sci. Res., Vol. 6, No. 3, pp. 37-42, 2017. 
[6] Y. Mae, "Correlation of the effects of alloying elements on the hardenability of steels to the diffusion coefficients of elements in Fe," Int. J. Mater. Sci. App., Vol. 6, No. 4, pp. 200-206, 2017.

[7] Japan Atomic Energy Research Institute, Data sheet JAERI $6010,1962$.

[8] http://en.wikipedia.org/wiki/Zirconium
[9] H. Sakurai, New knowledge about 111 elements, Koudan-sha, 1997, pp. 201.

[10] S. Nagakura, etc., Dictionary of physics and chemistry, Iwanami, 1998, p. 667.

[11] R. W. Bauer, J. D. Anderson, S. M. Grimes and V. A. Maden, "Application of Simple Ramsauer Model to Neutron Total Cross Section,” UCRL_JC_127199, 1997. 